\title{
Hypertension (Htn) among Young People of 18 to 35 Years Old in Cardiology Department of Gabriel Toure University Teaching Hospital
}

\author{
Ichaka Menta, Hamidou O. Ba, Kassoum M. Sanogo \\ Cardiology Department of “Gabrièl Touré” University Teaching Hospital, Bamako, Mali \\ Email: mentasomonosso@yahoo.fr, bhamiba@yahoo.fr
}

How to cite this paper: Menta, I., Ba, H.O. and Sanogo, K.M. (2018) Hypertension (Htn) among Young People of 18 to 35 Years Old in Cardiology Department of Gabriel Toure University Teaching Hospital. World Journal of Cardiovascular Diseases, 8, 11-17.

https://doi.org/10.4236/wjcd.2018.81002

Received: December 11, 2017

Accepted: January 8, 2018

Published: January 11, 2018

Copyright $\odot 2018$ by authors and Scientific Research Publishing Inc. This work is licensed under the Creative Commons Attribution International License (CC BY 4.0).

http://creativecommons.org/licenses/by/4.0/

\section{(c) (i) Open Access}

\begin{abstract}
Few studies have been conducted on hypertension among youth in Mali hence the interest of our study aimed to clarify certain aspects what are not taken account yet in previous studies. Objective: Determine the clinical and para-clinical characteristics of hypertension among 18 to 35 years old young people. Methods: This cross-sectional and retrospective study of 24 months from January $1^{\text {st }}, 2009$ to December $31^{\text {st }}, 2010$ in the cardiology department of the Gabriel Touré UTH. Results: We identified 132 cases during the study period out of 2146 cases of hypertension with a prevalence of $6.1 \%$. The female gender represented $81.8 \%$, with a sex ratio of $45.59,1 \%$ of patients had an unknown family history of hypertension. The age group of 31 to 35 years accounted for $43.9 \%$. The circumstances of discovery were dominated by exertional dyspnea (37.9\%). Body mass index was normal in $45.5 \%$ of patients. The higher BMI was more common in female patients with $39.39 \%$ ( $p=$ 0.045 ). In the sample, $53 \%$ of patients had a systolic blood pressure between 140 and $159 \mathrm{mmHg}$ and $38.6 \%$ had diastolic blood pressure $\geq 110 \mathrm{mmHg}$ with no significant difference $(p>0.5)$. The high creatinine level was found in $18.18 \%$ of our patients. The ECG found $84.6 \%$ of left ventricular hypertrophy among cavitary hypertrophies. The echocardiography has found cavitary dilatation in $40.04 \%$ of cases; it concerned the left ventricle in $25.71 \%$. Also $18.57 \%$ of our patients had impaired left ventricular systolic function. Among the complications found in our patients, kidney failure led with $56 \%$. Conclusion: Hypertension is not a rare event among young population even with a normal body mass index. It is often discovered during complications that can be dreadful.
\end{abstract}




\section{Keywords}

Hypertension, Young People, Complications

\section{Introduction}

Cardiovascular diseases still represent a major cause of morbidity and mortality. Young people are less likely than older people to believe that they have high blood pressure and then less likely to return to their doctor. Often, these are patients whose blood pressure would respond to weight loss and lifestyle changes, but they are less enthusiastic to seek treatment [1] [2].

In the world, many studies have concerned hypertension both in the elderly and in young adults. Thus we may note a prevalence of $12 \%$ in young medical students with an average age of 21 years in Argentina [3]. In Africa, the prevalence is generally higher in the adult population, $23 \%$ in South Africa, $15 \%$ in Cameroon, $31.1 \%$ in Tanzania and $31.4 \%$ in Zimbabwe [4].

This prevalence in West Africa in an adult population is estimated at $40 \%$ by some authors [5].

In Mali, estimates range from $16.6 \%$ to $45 \%$ [6] [7] [8]. However few studies have involved young people. We have initiated this study to identify clinical and complementary aspects of hypertension in the population from 18 to 35 years old in cardiology outpatient.

\section{Method}

The study was performed in the cardiology department of the Gabriel Touré UTH. This hospital because of its geographical location, is still the most visited hospital and is located in the administrative center of the city of Bamako.

This was a cross-sectional and retrospective study of 24 months from January $1^{\text {st }}, 2009$ to December $31^{\text {st }}, 2010$, over cardiology outpatients. The sample was composed of all patients during the study period who met these following inclusion criteria: Patients whose age was between 18 and 35 years, who agreed to participate in the study; in whom the diagnosis of hypertension was done after consultation and who carried out the first check-up including glucose blood test and creatinine blood test. The electrocardiogram and Doppler echocardiography were often made.

Hypertension was defined as blood pressure $\geq 140 / 90 \mathrm{mmHg}$.

Cardiac involvement was estimated by electrocardiogram and Doppler echocardiography.

The creatinine clearance was calculated using the formula of Cockroft and Gault:

$$
[(140 \text { - age }) \times \text { weight/creatinine: } \mathrm{mmol} / \mathrm{L}] \times k
$$

( $k=1.23$ for men, $k=1.04$ for women). It allowed us to evaluate the renal impact of hypertension. 


\begin{tabular}{ccc}
\hline RF stage & Description & GFR \\
G1 & Up and optimal & $>105$ \\
& & $90-104$ \\
G2 & Medium & $75-89$ \\
G3a & Medium to moderate & $60-74$ \\
G3b & Moderate to severe & $45-59$ \\
G4 & Severe & $30-44$ \\
G5 & ERF & $15-29$ \\
\hline
\end{tabular}

RF: Renal failure GFR: Glomerular filtration rate G: Grade ERF: End stage renal failure.

The body mass index was calculated using the following formula: $\mathrm{BMI}=$ weight/ height squared. Overweight was defined as BMI $\geq 25 \%$.

The diagnosis of stroke was made from the clinical examination and CT scan.

A survey questionnaire was developed for each patient. Data entry and analysis were performed on Microsoft WORD 2007 and SPSS 18.

\section{Results}

We recorded 132 cases of hypertension among 18 - 35 years old young people out of 2146 cases of hypertention with a frequency of $6.1 \%$. Patients whose age was between 31 and 35 years accounted for the majority, with $43.9 \%$ (Table 1). The females represented $81.8 \%$ with a sex ratio of 4.5 (Table 2).

Overweight was found in $48.5 \%$ of patients according to body mass index. The high BMI was more common in female patients with $39.39 \%(p=0.045)$. Hypertension was the main cardiovascular risk factor in our study with $39.4 \%$ followed by obesity with $20.4 \%$ and contraception with $15.2 \%$ (Table 3 ). Hypertension represented the most frequent personal history with $47.7 \%$. More than half of our patients had an unknown family history with $59.1 \%$. The circumstances of discovery were dominated by dyspnea with $37.9 \%$ (Table 4). In our sample, 53\% of our patients had a systolic blood pressure between 140 and 159 $\mathrm{mmHg}$ and $38.6 \%$ had a diastolic blood pressure $\geq 110 \mathrm{mmHg}$.

The standard electrocardiogram was performed in 103 of our patients with a rate of $78 \%$. The most frequent abnormality was left ventricular hypertrophy with $84.6 \%$. Doppler echocardiography was performed in 70 patients (53\%). It showed an alteration of the left ventricular systolic function in $18.57 \%$ of cases, and a dilatation of the same cavity in $25.71 \%$ of cases.

122 patients in the sample had performed a fasting glycaemia test and hyperglycemia was observed in $28.03 \%$. The creatinine clearance was abnormal in 31 of our patients by $23.5 \%$. Severe to end stage chronic renal failure was observed in $3.8 \%$ of our patients (creatinine clearance less than $30 \mathrm{~mL} / \mathrm{min}$ ). Among the complications found In 56 patients, the most frequent complication was the chronic renal failure (56\%) followed by heart failure (30\%) and stroke (5\%) (Table 5 and Table 6). 
Table 1. Distribution of patients according to age.

\begin{tabular}{ccc}
\hline Age groups & Numbers & Frequencies \\
\hline $18-20$ years & 11 & 8.4 \\
$21-25$ years & 18 & 13.6 \\
$26-30$ years & 45 & 34.1 \\
$31-35$ years & 58 & 43.9 \\
Total & 132 & 100.0 \\
\hline
\end{tabular}

Patients whose age is between 31 and 35 years make up the majority with $43.9 \%$.

Table 2. Distribution of patients according to gender.

\begin{tabular}{ccc}
\hline Gender & Numbers & Frequencies \\
\hline Male & 24 & 18.2 \\
Female & 108 & 81.8 \\
Total & 132 & 100.0 \\
\hline
\end{tabular}

Women accounted for $81.8 \%$, with a sex ratio of 4.5 .

Table 3. Distribution of patients according to their cardiovascular risk factors.

\begin{tabular}{ccc}
\hline Risk factors & Numbers & Frequencies \\
\hline Tobacco & 3 & 2.3 \\
Obesity & 27 & 20.4 \\
Hypertension & 52 & 39.4 \\
Diabetes & 3 & 2.3 \\
Sedentarity & 10 & 7.6 \\
Metabolic disorders & 10 & 7.6 \\
Stress & 7 & 5.3 \\
Unknown & 4 & 3.0 \\
Contraception & 20 & 15.2 \\
Corticosteroids & 1 & 0.8 \\
\hline
\end{tabular}

Hypertension was the main cardiovascular risk factor in our study with $39.4 \%$ followed by obesity with $20.4 \%$.

Table 4. Distribution of patients according to the circumstances of discovery.

\begin{tabular}{ccc}
\hline Circumstances of discovery & Numbers & Frequencies \\
\hline Fortuitous & 26 & 19.7 \\
Exertional dyspnea & 50 & 37.9 \\
Palpitations & 36 & 27.3 \\
Chest pain & 21 & 15.9 \\
Syncope & 1 & 0.8 \\
Lipothymia & 1 & 0.8 \\
Hydrops & 1 & 0.8 \\
Congestive heart failure & 1 & 0.8 \\
Nephropathy & 5 & 3.8 \\
Pregnancy & 15 & 11.4 \\
\hline
\end{tabular}

The circumstances of discovery were dominated by dyspnea with $37.9 \%$. 
Table 5. Distribution of patients according to the complications.

\begin{tabular}{ccc}
\hline Complications & Numbers & Frequencies \\
\hline Chronic renal failure & 31 & 56 \\
Heart failure & 17 & 30 \\
Stroke & 3 & 5 \\
Retinopathy & 3 & 5 \\
Pre-eclampsia & 1 & 2 \\
Angina & 1 & 2 \\
Total & 56 & 100 \\
\hline
\end{tabular}

In a total number of 56 patients showing complications the chronic renal failure led with $56 \%$.

Table 6. Distribution of patients according to creatinine clearance.

\begin{tabular}{ccc}
\hline Creatinine clearance $(\mathrm{ml} / \mathrm{min})$ & Numbers & Frequencies \\
\hline Advanced chronic renal failure $(10-15)$ & 3 & 2.3 \\
Severe chronic renal failure $(15-30)$ & 2 & 1.5 \\
Moderate chronic renal failure $(30-60)$ & 4 & 3.0 \\
Mild chronic renal failure $(60-100)$ & 22 & 16.7 \\
Normal Renal function $(>100)$ & 101 & 76.5 \\
Total & 132 & 100 \\
\hline
\end{tabular}

The creatinine clearance was abnormal for 31 of our patients (23.5\%).

\section{Discussion}

We found a hypertension frequency of $6.1 \%$ for 18 - 35 years age group and the age group between 31 and 35 years was mostly represented with $43.9 \%$. This result was close to $48.3 \%$ of Longo [9] for the 25 - 34 age group over a study on hypertension in Brazzaville.

Females predominated with $81.8 \%$ and the sex ratio was 4.5 . This result goes with the one of Diallo [7] (65\%), but in contrast with the one of Baragou [10] that reported male predominance (55\%). This difference could be explained by the fact that these authors are interested in severe and malignant hypertension in particular.

Being overweight is a risk factor consistently given by the authors [2] [3] [4] [7] [9] [10]. It was associated with $48.5 \%$ of patients with hypertension and represented $20.4 \%$ of risk factors. There was also a female predominance with $39.39 \%(p=0.045)$. This was reported by many authors, especially in black people living on the African continent or outside [2] [4] [7] [10] [11]. Some authors explain the female predominance by the impact of sociocultural factors on the one hand and on the other hand by the precarious living conditions of those living harsh lifestyle [2] [4].

Other cardiovascular risk factors were hypertension (39.4\%) and contraception (15.2\%). This high proportion of contraception, well above the $0.91 \%$ reported by Goeh Akue [12] in a Togolese series could be partly explained by the 
high incidence of women and their urban residence. Indeed, women accounted for $81.8 \%$ of the sample while they accounted for only $55.05 \%$ with a rural residence from the same author.

In the series $59.1 \%$ of patients did not know their family history. Goeh Akue [12] reported a family history rate of $7.34 \%$ and $45 \%$ from Diallo [7] in another study in Mali. $47.7 \%$ of patients recognized themselves as hypertensive before their care in cardiology, which result is below the $71 \%$ found by Kaba [13] and $68 \%$ from Baragou [10]. Another author [12] found a fortuitous discovery (52.75\%).

The circumstances of discovery were dominated by dyspnea with $37.9 \%$. This result is close to the $35.32 \%$ from Goeh Akue [12], higher than $13.45 \%$ from Baragou [10]. In the series $38.6 \%$ of patients had a diastolic blood pressure stage II of the JNCVII. This rate is lower than $73.4 \%$ [12] and 100\% [10] from some Togolese authors, and in accordance with $45.53 \%$ of Dembele [14].

Left ventricular hypertrophy on electrocardiogram was found in $84.6 \%$ of patients this rate is in disagreement with some authors [10] (21.76\%) [15] (24\%) and [4] (50\%). In addition heart failure with decreased systolic function was observed in $18.57 \%$, which is consistent with the $16 \%$ from some African series [4]. This is also consistent with the $19.75 \%$ from Dembéle [14].

$28.03 \%$ of patients have shown hyperglycemia which is higher than $6.47 \%$ [10] $2.13 \%$ [13] and $16.7 \%$ in internal medicine [14].

Chronic renal failure was noted in $23.5 \%$ of patients. This rate was $34 \%$ in a similar study in cardiology in Conakry [15] and $6.17 \%$ in internal medicine in Bamako [14]. This could be explained by a higher frequency of this kind of population as indicated by [2]. Some authors raise the issue of kidney as a victim or cause for hypertension [2] [15]. It seems that kidney disorders are the most common causes of hypertension in this population [2].

Beside renal failure $56 \%$ (with an end stage portion to $3.8 \%$ ), there were the heart failure (30\%) and stroke (5\%). These rates are consistent with the $55 \%$ of renal damage from Kaba [13], higher than $6.17 \%$ of renal failure and $19.75 \%$ of heart failure from Dembele [14]. Dembele has found a higher rate of stroke (12.34\%). This difference could be explained by the older age of these patients and also their confirmed diabetic status.

\section{The Limitations of the Study}

Our study has some limitations: the sample size (132 cases), no information on the fate of some of our patients with severe renal failure and also the absence of extensive investigations in these patients. All these difficulties seem to be related to the retrospective nature of the study on the one hand and on the other hand, the often high cost of these tests.

\section{Conclusion}

This study of 132 young people from 18 to 35 years old shows a very high frequency of hypertension and also a very high frequency of serious complications 
(kidney and heart failure, and stroke) in this very young population. This raises the problem of adequate care for young hypertensive in specialized centers. This health care reveals the issue of adherence and greater vigilance on screening for renal failure which is characterized by an early occurrence and rapid progression in this population.

\section{References}

[1] Akinkugbe, O. (1992) Pour une approche économique du traitement de l'HTA. Cardiol Trop, 69, 5-6.

[2] Steichen, O. (2010) Hypertension artérielle du sujet noir. Revue du praticien, 60, 654-659.

[3] Ennis, I.L., Gende, O.A. and Cingolani, H.E. (1998) Prevalence of Hypertension in 3154 Young Students. Medicina, 58, 483-491.

[4] Fourcade, L., Paule, P. and Mafart, B. (2007) Hypertension artérielle en Afrique subsaharienne: Actualité et perspectives. Médecine Tropicale, 67, 559-567.

[5] Cappuccio, F.P., Micah, F.B., Emmett, L., et al. (2004) Prevalence, Detection, Management, and Control of Hypertension in Ashanti, West Africa. Hypertension, 43, 1017-1022. https://doi.org/10.1161/01.HYP.0000126176.03319.d8

[6] Diallo, B.A. and Toure, M.K. (1994) Morbidité et mortalité cardio-vasculaire dans le service de cardiologie Bamako (Mali). Cardiologie Tropicale, 77, 21-25.

[7] Diallo, B.A. (1994) Profil épidémiologique de l'HTA en milieu hospitalier à Bamako. Médecine d'Afrique Noire, 41, 103-105.

[8] Conférence des Ministres de la Santé de l'UA (CAMH6) (1992) Incidence des maladies non transmissibles (MNT) et des maladies tropicales négligées (MTN) sur le développement en Afrique: État de l'hypertension en Afrique. Sixième session ordinaire, 22-26 avril 2013. Addis Abeba, ÉTHIOPIE.

[9] Longo, F., Melaman, S.F. and Tengoua, T.S. (2004) Données épidémiologiques sur l'hypertension artérielle et les autres facteurs de risque cardio-vasculaires des Brazzavillois: les dépêches de Brazzaville Mai 2004. Médecine d'Afrique Noire, 48, $1-5$.

[10] Baragou, R., Damorou, F., Afangnon, K., Goeh Akue, E. and Soussou, B. (1998) Les HTA sévères et malignes à la clinique cardiologique du CHU du campus de Lomé. Médecine d'Afrique Noire, 45, 587-591.

[11] Cooper, R., Rotimi, C., Ataman, S., et al. (1997) The Prevalence of Hypertension in Seven Populations of West African Origin. American Journal of Public Health, 87, 160-168. https://doi.org/10.2105/AJPH.87.2.160

[12] Goeh Akue, E., Yehadji, K.Y., Baragou, S., et al. (2008) Hypertension artérielle en milieu rural au Togo: cas du District Sanitaire d'Agou. Sciences et Médecine, 6, 65-69.

[13] Kaba, M.L., Balde, M.D., Bah, A.O., et al. (2005) Evaluation de l'atteinte rénale au cours de l'hypertension artérielle de l'adulte à Conakry. Médecine d'Afrique Noire, 55, 185-188.

[14] Dembélé, M., Sidibé, A.T., Traoré, H.A., et al. (2000) Association HTA-Diabète sucré dansle service de médecine internede l'hôpital du Point G Bamako. Sciences et Médecine, 47, 276-280.

[15] Kaba, M.L., Balde, M.D. and Bah, A.O. (2008) Atteinte rénale au cours de l'hypertension artérielle de l'adulte en milieu cardiologique à Conakry. MedAfr Noire, 55, 185-188. 